\title{
Crystal structure of caspase-11 CARD provides insights into caspase-11 activation
}

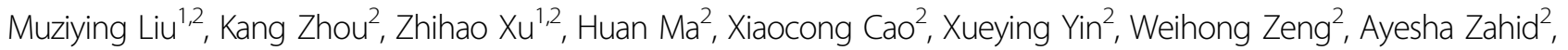
Sicheng $\mathrm{Fu}^{2}$, Kang $\mathrm{Ni}^{3}$, Xiaodong $\mathrm{Ye}^{3}$, Ying Zhou' ${ }^{1}, \mathrm{Li} \mathrm{Bai}^{2}$, Rongbin Zhou ${ }^{2,4}$ and Tengchuan Jin $\mathbb{B}^{1,2,4}$

\begin{abstract}
Murine caspase-11 is the centerpiece of the non-canonical inflammasome pathway that can respond to intracellular LPS and induce pyroptosis. Caspase-11 contains two components, an N-terminal caspase recruitment domain (CARD) and a C-terminal catalytic domain. The aggregation of caspase-11 is thought to promote the auto-processing and activation of caspase-11. However, the activation mechanism of caspase-11 remains unclear. In this study, we purified the caspase-11 CARD fused to an MBP tag and found it tetramerizes in solution. Crystallographic analysis reveals an extensive hydrophobic interface formed by the $\mathrm{H} 1-2$ helix mediating homotypic CARD interactions. Importantly, mutations of the helix $\mathrm{H} 1-2$ hydrophobic residues abolished the tetramerization of MBP-tagged CARD in solution and failed to induce pyroptosis in cells. Our study provides the first evidence of the homotypic interaction mode for an inflammatory caspase by crystal model. This finding demonstrates that the tetramerization of the N-terminal CARD can promote releasing of the catalytic domain auto-inhibition, leading to the caspase-11 activation.
\end{abstract}

\section{Introduction}

Inflammasome signaling pathways sense different body injuries and pathogen infections, and are essential components of the innate immune system ${ }^{1}$. Unlike several NOD-like receptors (NLRs) and AIM2-like receptors (ALRs) that, in response to certain Pathogen Associated Molecular Pattern (PAMPs), form a canonical inflammasome complex for activation caspase-1, the noncanonical pathway can directly respond to the cytosolic bacterial lipopolysaccharide (LPS), a molecule that plays an important role in endotoxic shock ${ }^{2-8}$.

Murine caspase- 11 and its human orthologs caspase- $4 / 5$ are essential for the non-canonical inflammasome pathway ${ }^{9}$. Sensing of cytoplasmic LPS results in the self-

\footnotetext{
Correspondence: Tengchuan Jin (jint@ustc.edu.cn)

'Department of Obstetrics and Gynecology, The First Affiliated Hospital of USTC, Division of Life Sciences and Medicine, University of Science and Technology of China, No.17 Lujiang Rd, Hefei, Anhui 230001, China ${ }^{2}$ Hefei National Laboratory for Physical Sciences at Microscale, CAS Key Laboratory of Innate Immunity and Chronic Disease, School of Basic Medical Sciences, Division of Life Sciences and Medicine, University of Science and Technology of China, Hefei, Anhui 230001, China

Full list of author information is available at the end of the article
}

cleavage and activation of caspase- $11^{10}$. The activated caspase can then drive the canonical inflammasome pathway to induce pyroptosis ${ }^{9,11-13}$. The activation of caspase-11 is involved in the development of inflammatory responses, such as lethal sepsis, making it an important target for drug development ${ }^{10}$.

Caspase-11, -4, and -5 belong to a family of aspartatespecific cysteine proteases. Their activation requires oligomerization and autoproteolysis. Caspase- $11,-4$, and -5 contain an $\mathrm{N}$-terminal death fold named caspase recruitment domain (CARD) and a C-terminal catalytic domain ${ }^{14}$. The CARD mediates caspase oligomerization through CARD-CARD interaction. Then, proximity-induced autoproteolysis separates the pro-domain from the catalytic domain (p30) and further separates the catalytic domain into a large and a small catalytic unit (p20 and p10), activating the caspase protein ${ }^{15,16}$. The CARD of caspase-11 was found to be essential for LPS-induced pyroptosis ${ }^{11}$. However, the structural mechanism of the caspase-11 CARD-CARD interactions remains unexplored.

Here we describe the crystal structure of caspase-11 CARD. The structure reveals that the caspase-11 CARD

\section{(c) The Author(s) 2020}

(c) (i) Open Access This article is licensed under a Creative Commons Attribution 4.0 International License, which permits use, sharing, adaptation, distribution and reproduction cc) in any medium or format, as long as you give appropriate credit to the original author(s) and the source, provide a link to the Creative Commons license, and indicate if changes were made. The images or other third party material in this article are included in the article's Creative Commons license, unless indicated otherwise in a credit line to the material. If material is not included in the article's Creative Commons license and your intended use is not permitted by statutory regulation or exceeds the permitted use, you will need to obtain permission directly from the copyright holder. To view a copy of this license, visit http://creativecommons.org/licenses/by/4.0/. 
undergoes tetramerization mediated by the hydrophobic interface in the H1-2 helix. Further mutagenesis experiments suggest that the hydrophobic interface is critical for the oligomerization of CARD and selfcleavage of caspase- 11 in cells.

\section{Results \\ MBP-tagged caspase-11 CARD exists as tetramers in solution}

The functional motif of caspase-11 CARD is reported to reside in amino acid residues $1-59$ of caspase-11 protein $^{11}$, although the canonical CARD region is predicted to be within residues $1-80$. To characterize the biochemical behavior, we generated an expression construct encoding the first 101 amino acids (aa1-101) of caspase11 with an maltose-binding protein (MBP) tag on the $\mathrm{N}$ terminus (WT-CARD ${ }^{\text {aa1-101 }}$ ). When purified from Escherichia coli, the WT-CARD ${ }^{\text {aal-101 }}$ with the MBP tag eluted with two peaks in a size-exclusion chromatogram (SEC). One peak represents polymers, probably resulting from misfolded samples, and the other represents a smaller sized species corresponding to a monomer (Fig. 1a). We further collected the protein samples in each peak to analyze their oligomerization states by static light scattering. The pooled monomer peak sample demonstrated a dynamic re-distribution between two different species (Fig. 1b, c), corresponding to tetramers and monomers (Fig. 1d). The continuous sedimentation coefficient distributions curves, $\mathrm{c}(\mathrm{s})$, for WT-CARD ${ }^{\text {aal-101 }}$ from monomer peak in SEC was obtained. The molecular weight of each peaks revealed a state switch from monomer to homodimer and homotetramer of WTCARD $^{\text {aa1-101 }}$, which also showed the state switch of WTCARD $^{\text {aal-101 }}$ was in a concentration-dependent manner (Fig. 1e). Interestingly, the deletion of the first ten amino acids on the $\mathrm{N}$ terminus led to a stable monomer of CARD $^{\text {aa11-101 }}$ in solution (Fig. 1c).

\section{Overall structure of the caspase-11 CARD in crystal}

The crystal structure of the caspase-11 CARD (residues 11-101) was determined at a resolution of $2.8 \AA$ using Xray crystallography with an N-terminal MBP as a crystallization tag (Table 1). This technique has been used successfully in the structure determination of several death domains ${ }^{17,18}$. As shown in Fig. 2a, the caspase-11 CARD contains four chains in one asymmetric unit (chain A, B, C, D). The structure of caspase-11 CARD displays a unique feature that is distinct from all known death domain superfamily members (Fig. 2a), it is composed of four amphipathic $\alpha$-helices instead of the classical six ${ }^{19}$. Comparison with the classic CARD structure reveals that, in the caspase-11 CARD, the classical helix $\mathrm{H} 1$ is fused with $\mathrm{H} 2$ to form a long helix that we named as $\mathrm{H} 1-2$. The three short helices following the $\mathrm{H} 1-2$ are well superimposable with the $\mathrm{H} 3, \mathrm{H} 4$, and $\mathrm{H} 5$ of classical death folds, but sequence alignment of the CARD domains shows that helices $\mathrm{H} 1$ to $\mathrm{H} 5$ are not highly conserved even among the homologous proteins (Fig. 2b, c). H6 cannot be observed in our crystal model (Fig. 2b).

The electrostatic surface analysis of the CARD with acidic, basic, and hydrophobic patches indicates that several neutral amino acids in the long H1-2 (residues L372-S391) form a hydrophobic surface, which possibly mediates the oligomerization of CARD (Fig. 2d). Moreover, the interactions between the four protomers are primarily mediated by H1-2 (Fig. 2e). PISA (Proteins, Interfaces, Structures, Assemblies) server ${ }^{20}$ was used to analysis the molecular interface in the structure. We found that the interface area between chain $B$ and chain $C$ is $1058.2 \AA^{2}$, whereas the area is $993.0 \AA^{2}$ between chain $\mathrm{A}$ and chain $\mathrm{D}$, with a negative $\Delta^{\mathrm{i}} \mathrm{G}$ indicating a strong hydrophobic interaction between two chains. Although the interface area reduced sharply between chain $\mathrm{A}$ and chain $B$, and between chain $C$ and chain $D$, the area is even less between chain $A$ and chain $C$, and between chain $B$ and chain $D$, suggesting that $B / C$ and $A / D$ formed homodimers before aggregating into a tetramer (Table 2). The presence of homodimer and homotetramer are also revealed by sedimentation velocity analytical ultracentrifugation (SV-AUC) analysis of WT-CARD ${ }^{\text {aa1-101 }}$ (Fig. 1e). All of these intermolecular interactions are primarily mediated by the H1-2 helix. Collectively, these data suggest the H1-2 of the caspase-11 CARD reveals by crystal structure might be involved in the self-interaction of caspase-11 CARD.

\section{Mutations in the hydrophobic interface abolish the aggregation of caspase- 11 in vitro}

To investigate whether the activation of caspase-11 relies on the homotypic interaction of CARD as observed in the crystal structure, we generated four mutations, V13A, L14A, L17A, V21A (VLLV) on the $\mathrm{H} 1-2$. These residues are all located in the N-terminal side of CARD domain in order to destroy the hydrophobic interaction (Fig. 3a). Compared with the charge mutation K19E, which positions in the middle of helix H1-2 (Fig. 3b) as mentioned in a previous study ${ }^{11}$, VLLV mutations on the caspase-11 CARD only change the side chains of the hydrophobic amino acids, but not their charges. C254A-caspase-11 containing the VLLV or K19E mutations in their H1-2s with the MBP tag, when purified from $E$. coli, were both eluted as monomers from the gel-filtration columns, whereas the MBPcaspase-11 protein carrying only the C254A mutation retained its ability to form oligomer with multiple polymerization states (Fig. 3c). This result demonstrates that the hydrophobic region of the CARD is required for the oligomerization of caspase- 11 . 

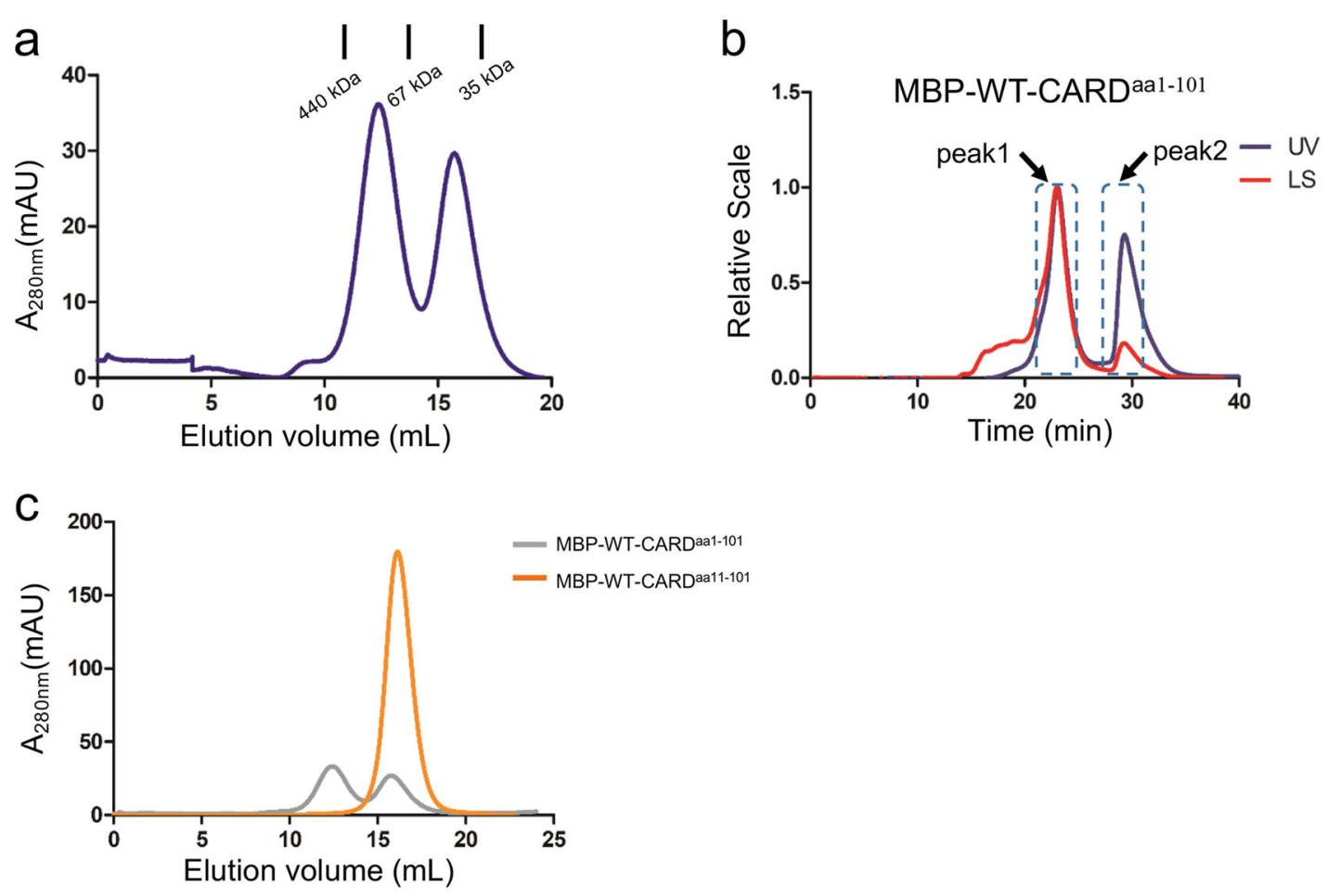

d

\begin{tabular}{ccc}
\hline Proteins(with MBP) & Peak1 MW(kDa) & Peak2 MW(kDa) \\
\hline MBP-WT-CARDaa1-101 & $223.6 \pm 1.9$ & $55.5 \pm 0.5$ \\
\hline
\end{tabular}

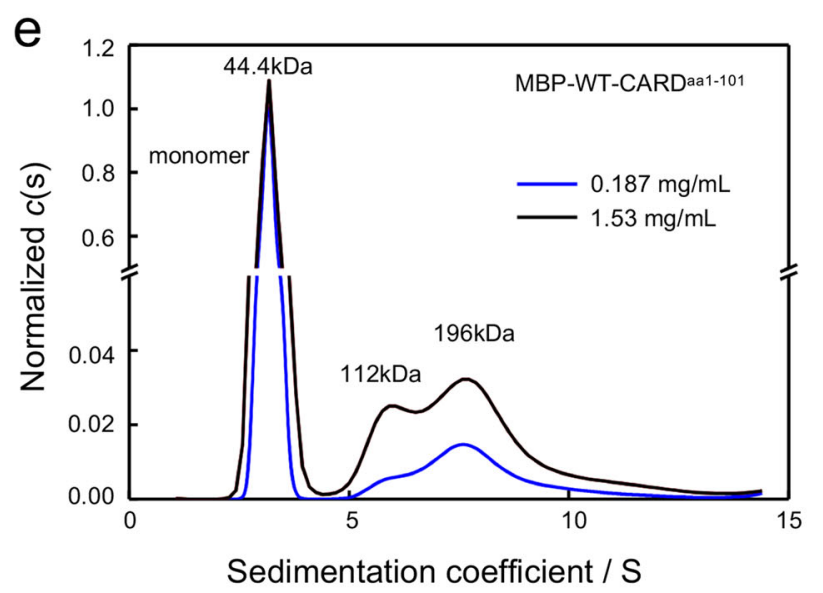

Fig. 1 Wild-type caspase-11 CARD is partially tetramerized in solution. a E. coli-expressed, MBP-tagged WT-CARD ${ }^{\text {aa } 1-101}$ protein elutes as two peaks, polymer and monomer. $\mathbf{b}$ Multi-angle light scattering (MALS) analysis of the monomer/oligomer peak. c Gel-filtration chromatography of WTCARD $^{\text {aa1-101 }}$ and WT-CARD ${ }^{\text {aa1 1-101 }}$ proteins (1 mg each). d ASTRA analysis of the molecular weights of different peaks in B. e Sedimentation velocity AUC profiles and the $c(s)$ distributions for WT-CARD ${ }^{\text {aal-101 }}$ (at $0.187,1.53 \mathrm{mg} / \mathrm{mL}$ ).

The abolished oligomerization ability in H1-2 mutant caspase-11 proteins prompted us to hypothesize that these mutations may also affect caspase-11 activation. A previous report showed that the wild-type caspase-11 protein failed to express in $E$. coli system ${ }^{13}$. Considering this, we used an MBP tag to stabilize caspase-11 in E. coli to investigate the caspase- 
Table 1 X-ray data collection and refinement parameters for caspase-11 CARD.

\begin{tabular}{|c|c|}
\hline No. of reflections (total/unique) & $302,171 / 51,251$ \\
\hline Space group & $\mathrm{P} 2{ }_{1} 2_{1} 2_{1}$ \\
\hline Redundancy ${ }^{a}$ & $5.89(5.77)$ \\
\hline \multicolumn{2}{|l|}{ Unit cell parameters } \\
\hline$a, b, c(\AA)$ & $77.31,145.16,187.72$ \\
\hline$a, \beta, \gamma\left(^{\circ}\right)$ & $90,90,90$ \\
\hline No. of measured reflections & $302,171(45,074)$ \\
\hline No. of unique reflections & $51,251(7804)$ \\
\hline Completeness $(\%)^{a}$ & $97.3(92.9)$ \\
\hline $1 / \sigma()^{a}$ & $20.64(2.03)$ \\
\hline R-means ${ }^{a}$ & $0.069(0.826)$ \\
\hline$C C(1 / 2)^{a}$ & $0.999(0.808)$ \\
\hline \multicolumn{2}{|l|}{ Refinement } \\
\hline Resolution $(\AA)$ & $50-2.8$ \\
\hline No. of protein atoms & 12,882 \\
\hline R.m.s.d. bond lengths $(\AA)$ & 0.011 \\
\hline R.m.s.d. bond angles $\left({ }^{\circ}\right)$ & 1.173 \\
\hline$R_{\text {work }}(\%)^{\mathrm{b}}$ & 30.55 \\
\hline$R_{\text {free }}(\%)^{c}$ & 34.01 \\
\hline Wilson B-factor & 84.15 \\
\hline Ramachandran plot (favored/disallowed) & $92.3 / 1.22$ \\
\hline PDB code & $6 K X G$ \\
\hline
\end{tabular}

Values in parentheses are for the highest-resolution shell.

${ }^{a} R_{\text {means }}=\Sigma_{h}(n / n-1)^{1 / 2} \Sigma_{i}\left|l_{i}(h)-\langle\mid(h)\rangle\right| / \Sigma_{h} \Sigma_{i} l_{i}(h)$, where $l_{i}(h)$ and $\langle\mid(h)\rangle$ are the ith and the mean measurement, respectively, of the intensity of reflection $h$.

${ }^{\mathrm{b}} R_{\text {work }}=\Sigma_{h}\left\|F_{\text {obs }}(h)|-| F_{\text {calc }}(h)\right\| / \Sigma_{h}\left|F_{\text {obs }}(h)\right|$, where $F_{\text {obs }}(h)$ and $F_{\text {calc }}(h)$ are the observed and calculated structure factors, respectively. No $1 / \sigma$ cutoff was applied.

${ }^{c} R_{\text {free }}$ is the $R$-value obtained from a test set consisting of a randomly selected $5 \%$ subset of the data. The test set is excluded from refinement.

11 activation. A $\sim 37 \mathrm{kDa}$ fraction was eluted from an analytical gel-filtration column and the fraction was further separated into two bands on an SDS gel (Fig. 3d). With $\mathrm{N}$-terminal sequencing and mass spectrum, we confirmed that the bands on the SDS gel were the p10 and p20 subunits of the caspase11 catalytic domain (Fig. 3e). The natural cleavage site of CARD is between the 59th and the 60th residue of caspase-11, and the two mature subunits of the catalytic domain (p30), p20 and p10, span residues 60-266, and 286-373, respectively (Fig. 3f). Activated caspase-11 cleaves gasdermin D (GSDMD) that can lead to pyroptosis and interleukin- $1 \beta$ maturation ${ }^{11,12}$. Therefore, to directly test the proteolytic activities in the wildtype and VLLV mutant caspase-11, we synthesized a selfquenched fluorescent peptide DABYL-GQLSLLSDGID-glu (edans) that mimics the GSDMD cleavage site for caspase-11.
This peptide can be used as a reporter for caspase-11 proteolytic activity (GSDMD cleavage reporter). We incubated auto-proteolytic wild-type p30 protein fraction with the GSDMD cleavage reporter. With the increased ratio of p30 to the GSDMD cleavage reporter, the fluorescent product increased, indicating that the fraction is proteolytically active (Fig. 3g). Then, we introduced the VLLV mutations to the wild-type caspase-11 and purified the protein from E. coli (VLLV-caspase-11). SDS-polyacrylamide gel electrophoresis analysis showed that the VLLV mutation reduced the selfcleavage of full-length caspase-11 (Fig. 3h). Furthermore, we found that the proteolytic activity of VLLV-caspase-11 was greatly reduced (Fig. 3i). These results show that the VLLV mutation on CARD not only affect the aggregation of caspase11 but also inhibit the cleavage of caspase- 11 itself and substrates.

Together, these data indicate that the hydrophobic interface on the CARD helix H1-2 region is essential for the aggregation of caspase- 11 that leads to caspase maturation and downstream signal cascade activation.

\section{Caspase-11-mediated GSDMD cleavage and cell death are} dependent on the hydrophobic surface on H1-2 of CARD

As we found that purified VLLV-caspase-11 fails to activate, we further sought to evaluate the activity of wildtype and mutant caspase- 11 in cells. As caspase- 4 is not expressed in HEK293T cells (Supplementary Fig. S1), we co-transfected hGSDMD with full-length caspase-11 plasmids, i.e., WT-caspase-11, VLLV-caspase-11, K19Ecaspase-11, or C254A-caspase-11, into HEK293T cells. Without any stimulation, WT-caspase-11 underwent selfactivation, then the hGSDMD was cleaved into two fragments (Fig. 4a). In addition, cell death was significantly upregulated (Fig. 4b). In summary, compared to WT-caspase-11, C254A-caspase-11 displayed a drastic decrease in the hGSDMD cleavage and cell death, and the VLLV-caspase-11 mutant displayed a partial decrease in both GSDMD cleavage and cell death.

We further tested whether the overexpressed wild-type and mutant caspase-11 could nucleate oligomeric assemblies of the caspase-11 protein. For this, we constructed wild-type and mutant caspase-11 expression plasmids tethered with enhanced green fluorescent protein (EGFP) on the $C$ terminus (WT-caspase-11-EGFP, VLLV-caspase-11-EGFP, and C254A-caspase-11-EGFP). When transfecting 293T cells with WT-caspase-11-EGFP or C254A-caspase-11-EGFP, we observed caspase-11EGFP specks that indicate caspase- 11 aggregations. In comparison, significantly less percentage of cells transfected with the VLLV-caspase-11-EGFP had caspase-11EGFP specks (Fig. 4c, d). These results demonstrate that the caspase-11-CARD mutations can affect the oligomerization and the activation of caspase- 11 in cells. 
a
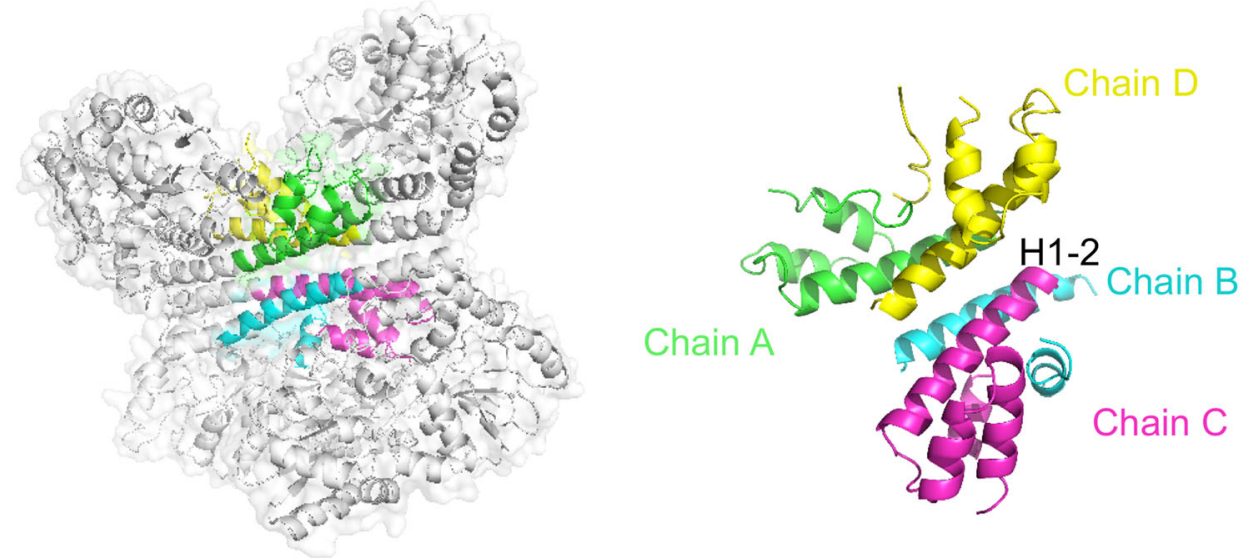

b

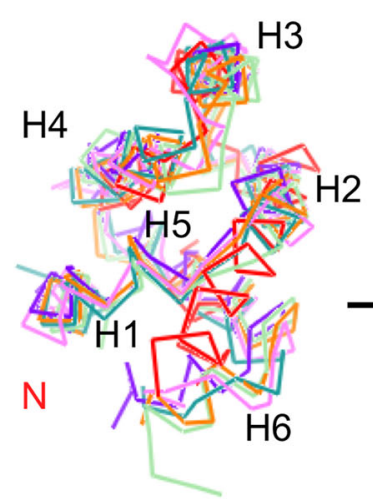

C

H1-2 helix

H3
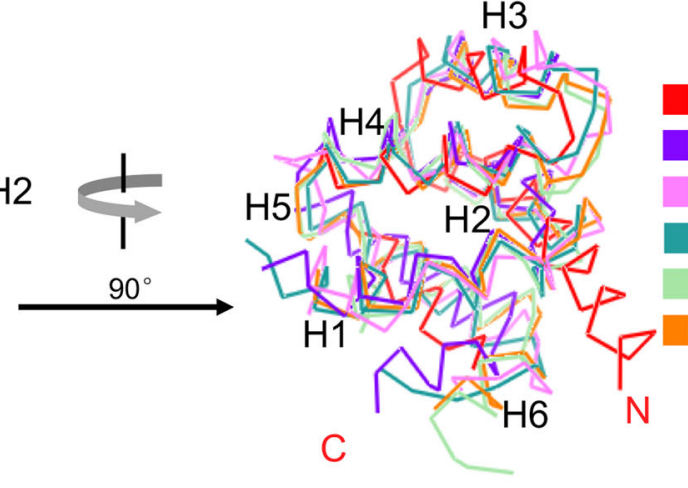

caspase-11 CARD

CARMA1 CARD

caspase-1 CARD

RIP2 CARD

ASC CARD

NLRC4 CARD

Caspase-11

$1 \quad 10$ elecelecelecelecel

H3 helix

H4 helix

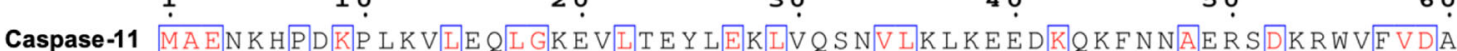

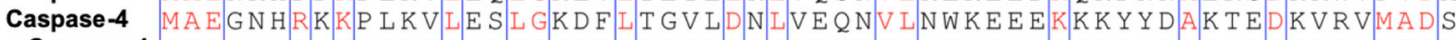
mCaspase-1 M A D K I L R A

hCaspase-1 MADKVLKEKRKLFIRSMGEGTINGL LDELLQTRVLNKEEMEKVKRENATVMDK T RAL IDS

ASC

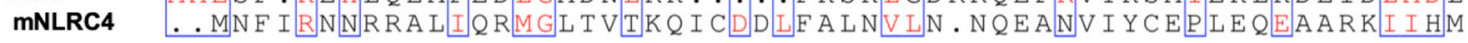

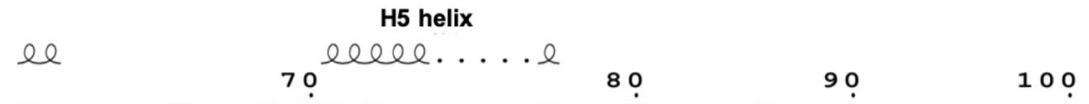

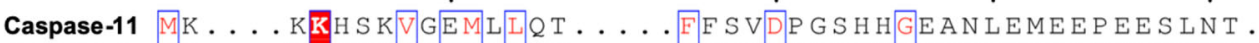

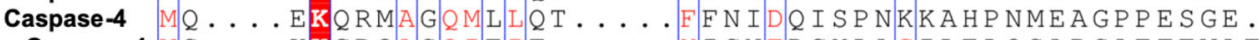

mCaspase-1 VIS ....KKG P Q A S Q IFIT . . . . . Y I CNED C Y LAGI LELQS APSAETFVA T

hCaspase-1 VI ..... PKGA QA C Q ICIIT ...... Y I CEED S Y LAG T L G L SADQT S GNY LNM

ASC $\quad$ MVGVFTSKDAVSVTVEILRAIKCNAVADDILRNTGQSESKGALSDES . .

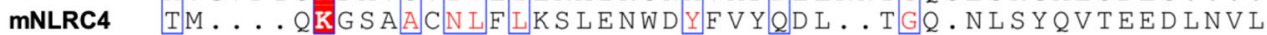

d
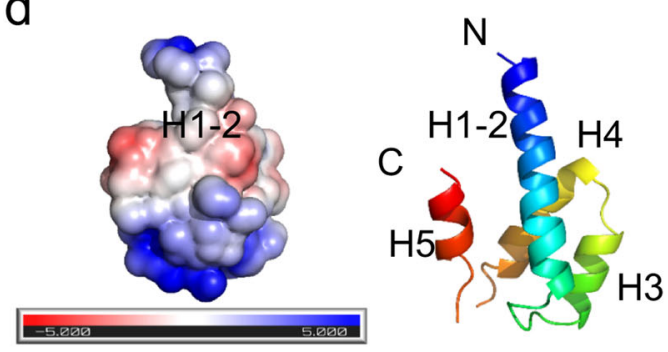

e
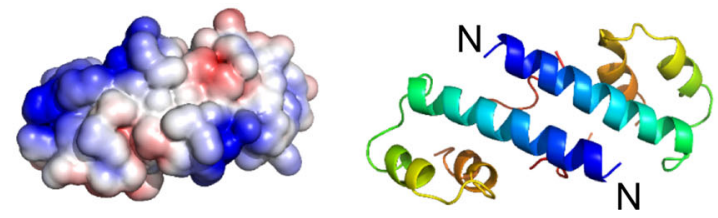

Fig. 2 (See legend on next page.) 
(see figure on previous page)

Fig. 2 Overall structure of caspase-11 CARD. a Homotetrameric packing of caspase-11 CARD in crystal. The H1-2 helices reside in the center of the interaction interfaces. b A stereo diagram of superimposed known CARD structures: CARMA1-CARD (PDB ID: 4jup), caspase-1-CARD (PDB ID: 5fna), RIP2-CARD (PDB ID: 6ggs), ASC-CARD (PDB ID: 5gpq), NLRC4-CARD (PDB ID: 6n1i), and caspase-11 CARD. c Sequence alignment of residues 1-101 of caspase-11, caspase-4, mcaspsae-1, hcaspase-1, ASC, and mNLRC4. Different amino acids are in white and conserved residues are highlighted in red. The secondary structure of caspase-11 CARD is shown above the sequence. $\mathbf{d}$ An electrostatic surface of Chain C generated with APBS software. Negatively charged regions are represented in red, neutral regions in white, and positively charged regions in blue. Locations of the hydrophobic interface are indicated. The $\mathrm{N}$ terminus sequence of $\mathrm{H} 1-2$ contains neutral amino acids (white). The direction of the chain ( $\mathrm{N}-\mathrm{C}$ terminus) is colored as blue to red. e An electrostatic surface representation of Chain A and D generated with APBS software. Negatively charged regions are represented in red, neutral regions in white, and positively charged regions in blue. Locations of the hydrophobic interface are indicated. For each chain, the $\mathrm{N}$ terminus sequence of $\mathrm{H} 1-2$ contains neutral amino acids (white). The direction of the chain ( $\mathrm{N}-\mathrm{C}$ terminus) is colored as blue to red.

\section{LPS does not associate with the MBP-tagged caspase-11 CARD domain in solution}

The above results showed that the homotypic CARD interaction interface is critical for caspase-11 activation and overexpressing caspase-11 proteins in HEK293T cells could directly activate the non-canonical inflammasome signaling pathway without LPS stimulation through CARD-mediated oligomerization of caspase-11. Previous research suggests that the LPS binds to the CARD domain of caspase-4, which promotes caspase-4 activation ${ }^{11}$, but another research indicates that LPS binding requires both CARD and catalytic domain of caspase- $11^{21}$. We next tested whether LPS is required for the aggregation of caspase-11 CARD in solution. The deletion of residues 1-59 of caspase-11 was shown to compromise the LPS-induced caspase-11 activation ${ }^{11}$. Here we used the MBP fusion protein to stabilize the caspase-11 CARD and the mutant VLLV-CARD in the E. coli expression system, with a $\sim 10$ residue linker (TEV cleavage sequence) between MBP and caspase-11 CARD proteins. Caspase-11 CARD ${ }^{\text {aa11-101 }}$ and VLLV-CARD ${ }^{\text {aa11-101 }}$ formed monomers during purification, which was confirmed by SEC-multi-angle light scattering (MALS) (size-exclusion chromatography followed by MALS (Fig. 5a, b). After incubation with LPS at $4{ }^{\circ} \mathrm{C}$ for $4 \mathrm{~h}$, the gel-filtration profile displayed no change (Fig. 5c).

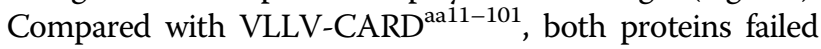
to respond to LPS stimulation (Fig. 5d). Similar to WTCARD $^{\text {aa11-101 }}$ and VLLV-CARD ${ }^{\text {aa11-101 }}$, LPS failed to induce the polymerization of WT-CARD ${ }^{\text {aa1-101 }}$ and VLLVCARD $^{\text {aa1-101 }}$ (Fig. 5e, f). These data led to the conclusion that LPS does not directly bind with MBP-tagged caspase11 CARD, despite that it is able to form functional related homo-oligomers.

\section{Truncations of caspase-11 CARD result in different dynamic state and which is in a linker sequence independent manner}

For crystallization, we used N-terminal 10 amino acids deficient CARD (11-101 residues), but it stays as monomer in solution during purification (Fig. 1d). To find out whether CARD aggregation requires all of the residues in the CARD domain, we made several truncations of WT-
CARD, WT-CARD ${ }^{\text {aa1-59 }}$, and WT-CARD ${ }^{\text {aa11-59 due to the }}$ natural cleavage site of caspase-11 (Fig. 3e). Unfortunately, we failed to obtain the crystal structure of different truncations of CARD. Therefore, we decided to determine the mechanism of oligomerization of CARD based on their structural characteristics. WT-CARD ${ }^{\text {aa1-101 }}$ showed a tetrameric state in solution during purification (Fig. 1a, b, d). Further, we also detected VLLV-CARD ${ }^{\mathrm{aa} 1-101}$, similar to WT-CARD ${ }^{\text {aal-101 }}$, demonstrates an equilibrium between oligomers and monomers during gel-filtration purification (Fig. 6a, b), even though unlike WT-CARD ${ }^{\text {aa1-101, the }}$ monomeric state appears to dominate for VLLVCARD $^{\text {aa1-101 }}$. Among different truncations, only WT-

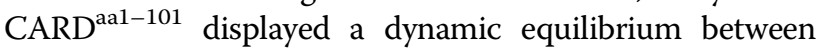
tetramers and monomers, whereas other truncations are monomers (Fig. 6c). Therefore, we hypothesized that the first ten residues are critical for its self-association when fused at the $\mathrm{C}$ terminus of MBP. To test whether the sequence of the first ten amino acids is required for CARD self-assembly, we replaced the first ten amino acids to a flexible GS repeat sequence (5'-GSGSGSGSGS-3') in WTCARD $^{\text {aa1-101 }}$. Compared with WT-CARD ${ }^{\text {aal-101 }}$, the GS repeat mutant showed no change in the tetramer peak (Fig. 6e). This suggests that a flexible loop at the $\mathrm{N}$ terminus, regardless of the sequence, is required for the oligomerization of CARD.

We also sought to find out the resting mechanism of caspase-11. We further hypothesized that there might exist some self-interaction between CARD and catalytic domain of caspase-11. Unfortunately, we failed to observe the interaction of the two units of caspase-11 (Fig. 6f). This result indicated there might be some more subtle mechanism of self-inhibition for caspase-11.

\section{Discussion}

We determined, for the first time, the crystal structure of the CARD domain in an inflammatory caspase. Our data show that the structure of caspase-11 CARD forms an atypical conformation different from other death domain superfamily members. And this conformation indicates a possible activation model of caspase-11 by the hydrophobic interaction mediated by CARD H1-2 (Fig. 7). 
Table 2 PISA analysis of the four chains in the crystal structure.

\begin{tabular}{lccc}
\hline Structure1 : Structure2 & Interface area $\left(\AA^{2}\right)$ & $\Delta^{\mathrm{i}} \mathbf{G}(\mathbf{k c a l} / \mathbf{m o l})$ & $\boldsymbol{\Delta}^{\mathrm{i} G} \boldsymbol{P}$-value \\
\hline Chain C: Chain B & 1058.2 & -18.7 & 0.361 \\
Chain A: Chain D & 993.0 & -20.2 & 0.226 \\
Chain A: Chain B & 241.0 & -5.8 & 0.296 \\
Chain C: Chain D & 224.9 & -4.8 & 0.210 \\
Chain D: Chain B & 83.1 & -2.7 & 0.143 \\
Chain A: Chain C & 82.1 & -2.6 & 0.093 \\
\hline
\end{tabular}

$\Delta^{\mathrm{i} G}$ indicates the solvation free energy gain upon formation of the interface, in $\mathrm{kcal} / \mathrm{mol}$. Negative $\Delta^{\mathrm{i}} \mathrm{G}$ indicates hydrophobic interfaces or positive protein affinity. This value does not include the effect of the satisfied hydrogen bonds and salt bridges across the interface.

$P>0.5$ means that the interface is less hydrophobic than it should be, therefore the interface is likely to be an artifact of crystal packing. $P<0.5$ indicates the interface has a higher hydrophobicity than the predicted average for a given structure, implying that the interface surface may be interaction-specific. The limiting case of $P=0$ means that no other interface of the observed area may have a lower $\Delta^{i} G$; therefore, this interface is a truly unique spot on the protein surface.

Death fold is generally packed loosely ${ }^{19}$ and several death domain members have non-classic structures during activation. For example, DR3 DD H3 and H4 undergo a conformation switch when interacting with tumor necrosis factor receptor-associated death domain ${ }^{17}$; FAD binding can induce the shift of helix $\mathrm{H} 6$ to $\mathrm{H} 5$ in Fasassociated protein with death domain, which forms a stem helix for the adaptor binding ${ }^{22}$. Therefore, the conformational changes of death domains are often associated with biological functions.

The constructs of caspase-11 CARD and full-length protein both with all mutants are linked with MBP on Nterminal side and His-tag on C-terminal side. The linker (VD) between MBP and the target protein was reported to have minimal impact on the structural and functional characteristics of the target protein ${ }^{23}$. Also, the linker brings the MBP tag closer to the CARD, which can stabilize the protein in the crystal (Supplementary Fig. S2). If removing the MBP tag after purification, we failed to obtain WT-CARD ${ }^{\text {aa11-101 }}$ (data not shown). In contrast, the mutant VLLV-CARD ${ }^{\text {aa11-101 }}$ is more stable in solution: it can be purified when the MBP tag in the $\mathrm{N}$ terminus is removed using TEV protease (Supplementary Fig. S3).

Inflammatory caspases (caspase-1/4/5/11) all contain a CARD and a catalytic domain ${ }^{14,24}$. Oligomerization caused by CARD-CARD interactions leads to self-cleavage, activation of the catalytic domain, cleavage of GSDMD, and the induction of pyroptosis. Our data show that the homotypic CARD-CARD interface mediated by hydrophobic residues in the elongated $\mathrm{H} 1-2$ is critical for the oligomerization of caspase- 11 and its activation. Mutations on $\mathrm{H} 1-2$ could inhibit the aggregation of caspase-11 and reduce cell death induced by caspase- 11 . In addition, our results showed that LPS failed to bind with the MBPtagged caspase-11 CARD. Maybe there also exist an intermediate molecular interaction that directly bind with caspase-11 in response to intracellular LPS stimulation or
LPS only educated caspase- 11 and then lead to activation. The N-terminal 1-59 residues of caspase-11 were reported to be critical for the activation of caspase- 11 induced by LPS $^{11}$. Our study further pinpointed that the H1-2 helix is essential for the oligomerization of caspase-11 during activation. This study of caspase-11 CARD structure and activation mechanism may provide insights into understanding the mechanism of inflammatory caspases in general and future drug development that aims to inhibit inflammasome activation.

Due to technical restrictions, we failed to solve the structure of full-length caspase-11 in resting state. In addition, the exact oligomerization state of full-length caspase-11 remains unknown. It is worth investigating whether the tetramer state observed in our CARD structure is maintained in full-length protein setting.

\section{Materials and methods \\ Plasmids and transfection}

Complementary DNA (cDNA) of murine caspase-11 was amplified from the plasmids provided by Feng Shao. The mutant and wild-type caspase-11 cDNAs were inserted into a pEGFP-N1 vector for transient expression in HEK293T cells. A modified pCDNA3.1 vector was used to co-express GSDMD with mutant and wild-type caspase-11 in HEK293T cells. All plasmids were verified by DNA sequencing.

\section{Purification of recombinant proteins}

E. coli Rosetta and BL21 (DE3) cells harboring the V28E-CARD plasmid (MBP vector with an N-terminal MBP tag and a C-terminal His6-tag) were grown in LuriaBertani Broth (LB broth) medium supplemented with $50 \mu \mathrm{g} / \mathrm{mL}$ kanamycin. Protein expression was induced for $4 \mathrm{~h}$ at $18^{\circ} \mathrm{C}$ with $0.3 \mathrm{mM}$ isopropyl- $\beta$-D-thiogalactopyranoside after $\mathrm{OD}_{600}$ reached 1.2. The cells were collected by centrifugation and lysed by ultrasonication in a lysis buffer containing $150 \mathrm{mM} \mathrm{NaCl}, 20 \mathrm{mM}$ imidazole, and 


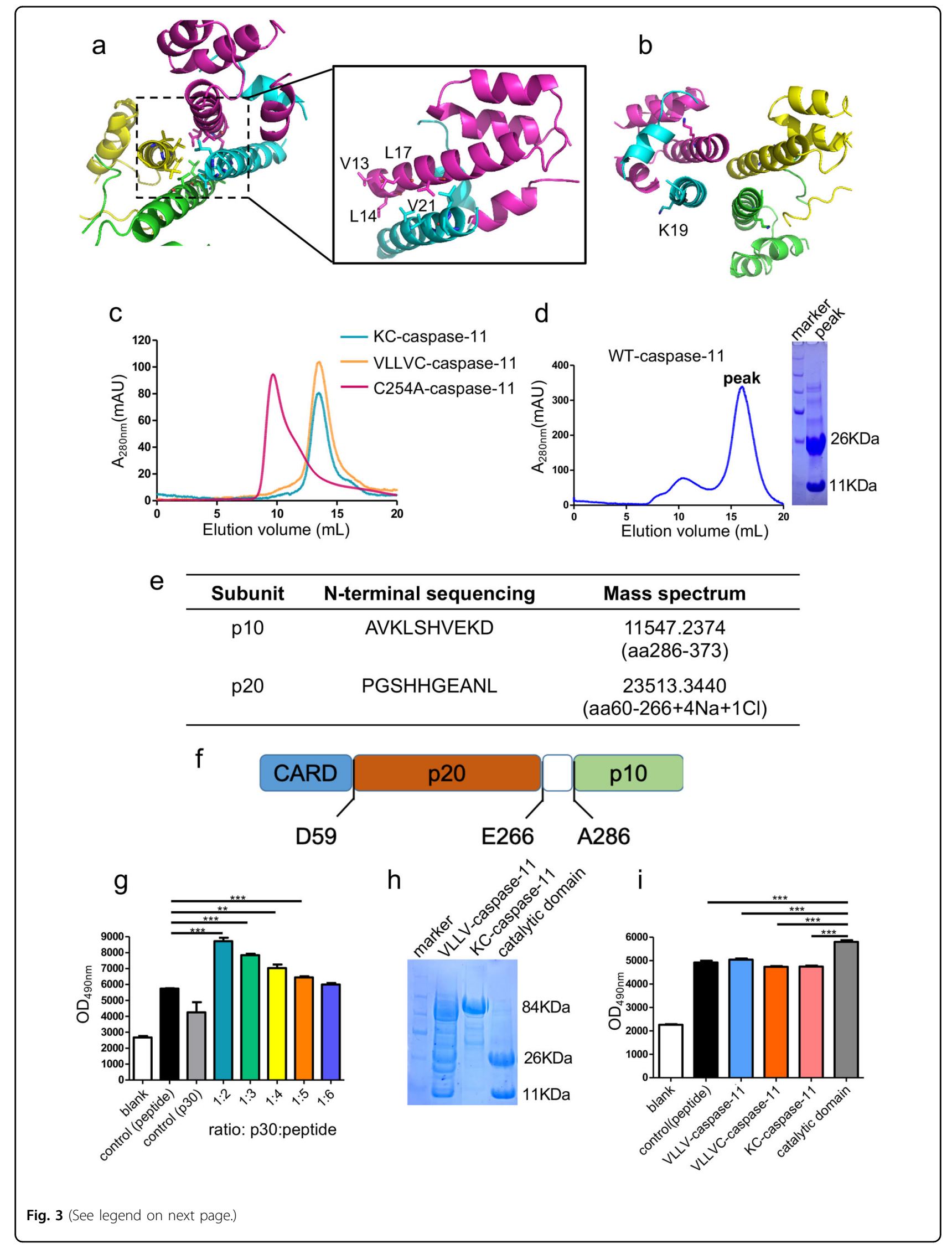


(see figure on previous page)

Fig. 3 Mutations abolish the aggregation of caspase-11 CARD and prohibit the self-cleavage of caspase-11. a The four hydrophobic amino acids on $\mathrm{H1}-2, \mathrm{~V} 13, \mathrm{~L} 14, \mathrm{~L} 17$, and V21, are indicated as sticks. $\mathbf{b}$ The basic amino acid K19 resides in the middle of H1-2 and is indicated as a stick. c Gel-filtration chromatography of E. coli-expressed C254A-caspase-11, K19E-caspase-11, and VLLV-caspase-11 (both in K19E-caspase-11 and VLLVcaspase-11 are on the C254A background). $\mathbf{d}$ E. coli-expressed wild-type mature caspase-11 protein (left panel), the corresponding SDS-PAGE gel is shown on the right. e Initial ten amino acids detected by N-terminal sequencing of the two bands in $\mathbf{d}$ transferred to a PVDF membrane and mass spectrum detecting the molecular weight of the two bands in $\mathbf{d}$. $\mathbf{f}$ Diagram of caspase-11 and the self-cleavage sites. $\mathbf{g}$ Proteolytic activity assay on the predicted caspase-11 catalytic domain. GSDMD cleavage reporter was mixed with different amounts of the predicted caspase-11 catalytic domain. The initial concentration of predicted caspase-11-catalytic domain is $1 \mu \mathrm{M}$. All data shown are representative of at least three independent repeats. $\mathbf{h}$ VLLV mutation reduces self-cleavage of full-length caspase-11 revealed by SDS-PAGE analysis. i Synthetic DABYL-GQLSLLSDGID-glu (edans) (GSDMD cleavage reporter) was used as the substrate of mature caspase-11 and the concentration ratio of caspase-11 proteins to the reporter is $1: 5$. All data shown are representative of at least three independent repeats.
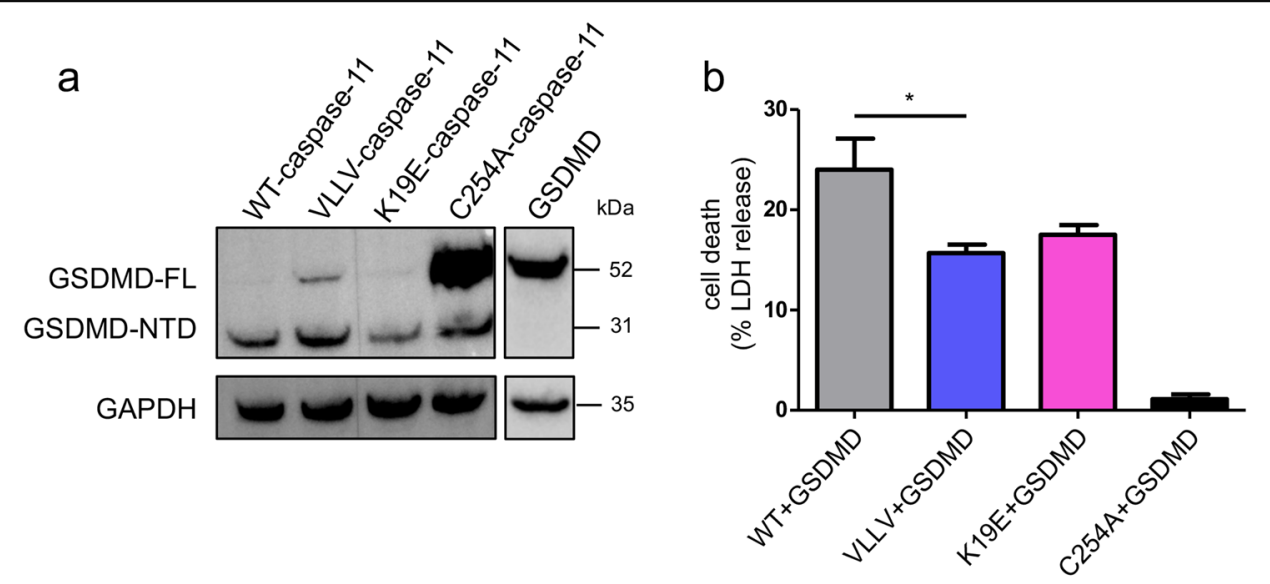

C

WT-caspase-11 VLLV-caspase-11 C254A-caspase-11
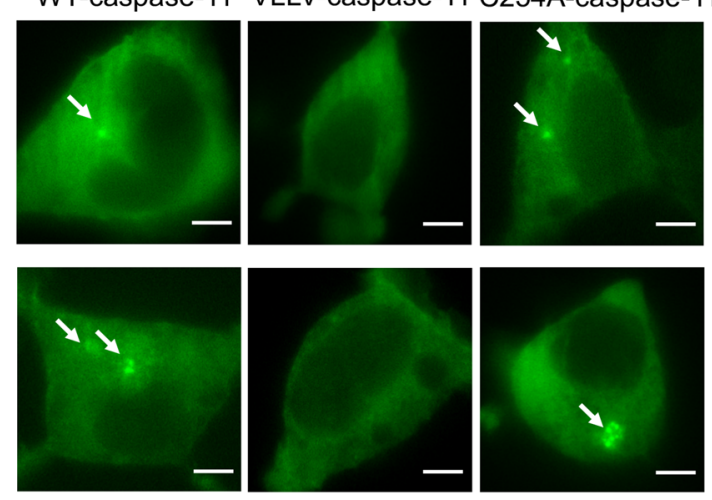

d

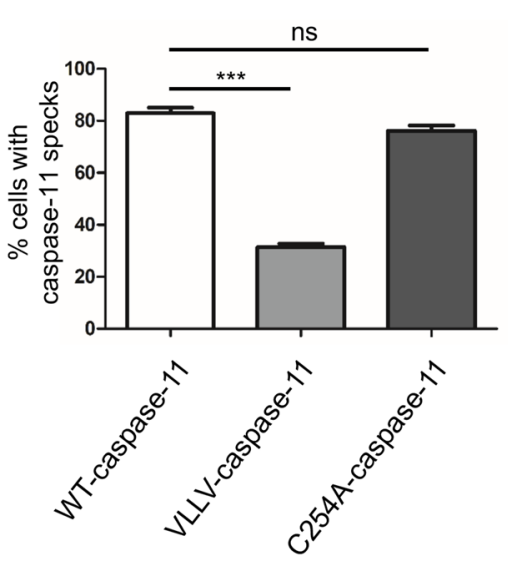

Fig. 4 The hydrophobic interface is critical for the cellular activation of the caspase-11 inflammasome. a Immunoblot analysis of GSDMD and GAPDH in the HEK293T cell lysates after $24 \mathrm{~h}$ of transfection with caspase-11 and GSDMD. b Same experiment as in a, cell death was monitored by LDH release in HEK293T cell. Data are shown as mean \pm SEM $(* P<0.05$, two-tailed $t$-test). c Caspase-11-EGFP speck formation (arrows) in HEK293T cells at $24 \mathrm{~h}$ post transfection (scale bars $=5 \mu \mathrm{m}$ ). d Quantitative comparison of caspase-11-EGFP speck formation in c. Quantified values are shown as mean \pm SEM ( $\geq 50$ cells per group were pooled from three independent experiments. ${ }^{*} P<0.05$, ${ }^{*} P<0.01$, and ${ }^{* * *} P<0.001$, two-tailed t-test).

$20 \mathrm{mM}$ Tris- $\mathrm{HCl}(\mathrm{pH} 8.0)$. The cell lysate was clarified by centrifugation at $15,000 \times g$ at $4{ }^{\circ} \mathrm{C}$ for $20 \mathrm{~min}$ and residual debris was removed using a $0.8 \mu \mathrm{m}$ filter. The supernatant containing the target protein was loaded onto a nickel column (GE Healthcare Life Sciences). The recombinant protein was eluted using the lysis buffer supplemented with $400 \mathrm{mM}$ imidazole. The His6-MBP-tagged protein was further purified through Superdex 200 gel-filtration chromatography (GE Healthcare Life Sciences) in a buffer containing $250 \mathrm{mM} \mathrm{NaCl}$ and $10 \mathrm{mM}$ Tris- $\mathrm{HCl}(\mathrm{pH} 8.0)$. 

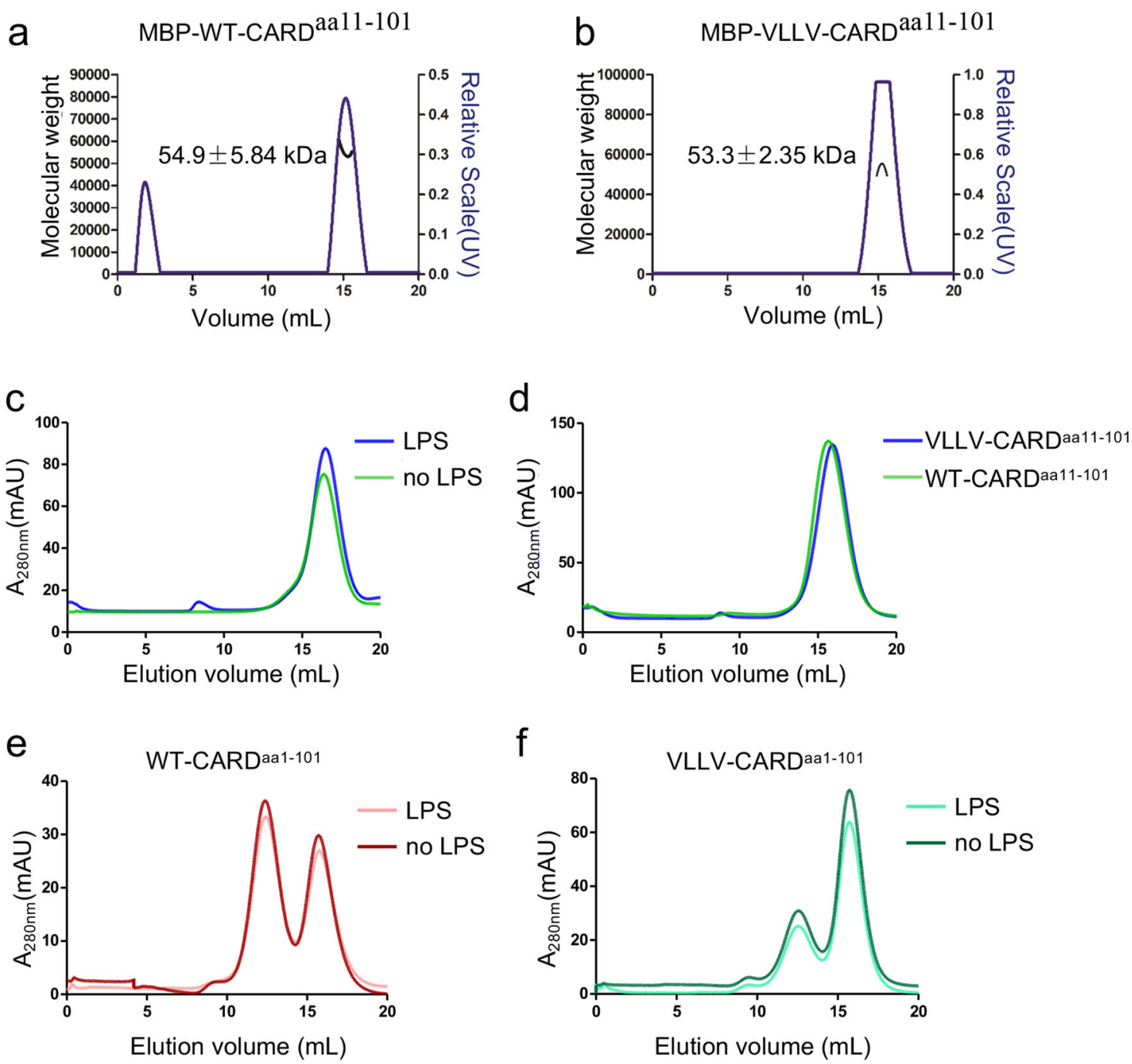

Fig. 5 LPS fails to induce the aggregation of wild-type and mutant caspase-11 CARDs. a, b Multi-angle light scattering (MALS) profile. The black line indicates the experimentally calculated molecular weight. c Purified caspase-11 WT-CARDaa11-101 protein with a TEV recognition sequence between MBP fusion protein and CARD. Protein $(1 \mathrm{mg} / \mathrm{mL})$ was incubated with $5 \mu \mathrm{g}$ LPS at $4{ }^{\circ} \mathrm{C}$ for $4 \mathrm{~h}$. d Purified WT-CARD aa11-101 and VLLVCARD ${ }^{\text {aa1 1-101 }}$ with MBP tag (1 mg each) incubated with $5 \mu \mathrm{g}$ LPS at $4^{\circ} \mathrm{C}$ for $4 \mathrm{~h}$. e, f Purified WT-CARD ${ }^{\text {aal-101 }}$ and VLLV-CARD ${ }^{\text {aal-101 }}$ with MBP tag (1 mg each) incubated with or without $5 \mu \mathrm{g}$ LPS at $4{ }^{\circ} \mathrm{C}$ for $4 \mathrm{~h}$.

Crystallization, X-ray diffraction, structure determination, and refinement

Purified MBP-tagged wild-type CARD protein was concentrated using Amicon centrifugal concentrators (Millipore) to $85 \mathrm{mg} / \mathrm{mL}$. Then, hanging drops were set up for vapor diffusion crystallization. The protein was crystallized at $291 \mathrm{~K}$ for a week with a well solution containing 2.4 $\mathrm{M}$ ammonium sulfate and 5\% isopropanol. Ethylene glycol was added to the reservoir solution to $20 \%$ $(\mathrm{v} / \mathrm{v})$ as the cryoprotectant and the crystals were flashcooled in liquid nitrogen for X-ray diffraction data collection.

X-ray diffraction data were collected at the Shanghai Synchrotron Radiation Facility beamline BL17U1 ${ }^{25}$. Data were processed with the HKL2000 program suite ${ }^{26}$ and $\mathrm{XDS}^{27}$. The structure was determined using molecular replacement method and refined with the Phenix program suite $^{28}$. The MBP structure from the Protein Data Bank (PDB ID: 4IRL) was used as the search model ${ }^{29}$. Model building was performed using Coot (Crystallographic Object-Oriented Toolkit). The structure was validated using the Molprobity server and the RCSB ADIT validation server before deposition. Figures were produced using the program Pymol (Schrödinger).

\section{Antibodies and reagents}

GSDMD antibody was obtained from Sigma-Aldrich (G7422). Glyceraldehyde 3-phosphate dehydrogenase 

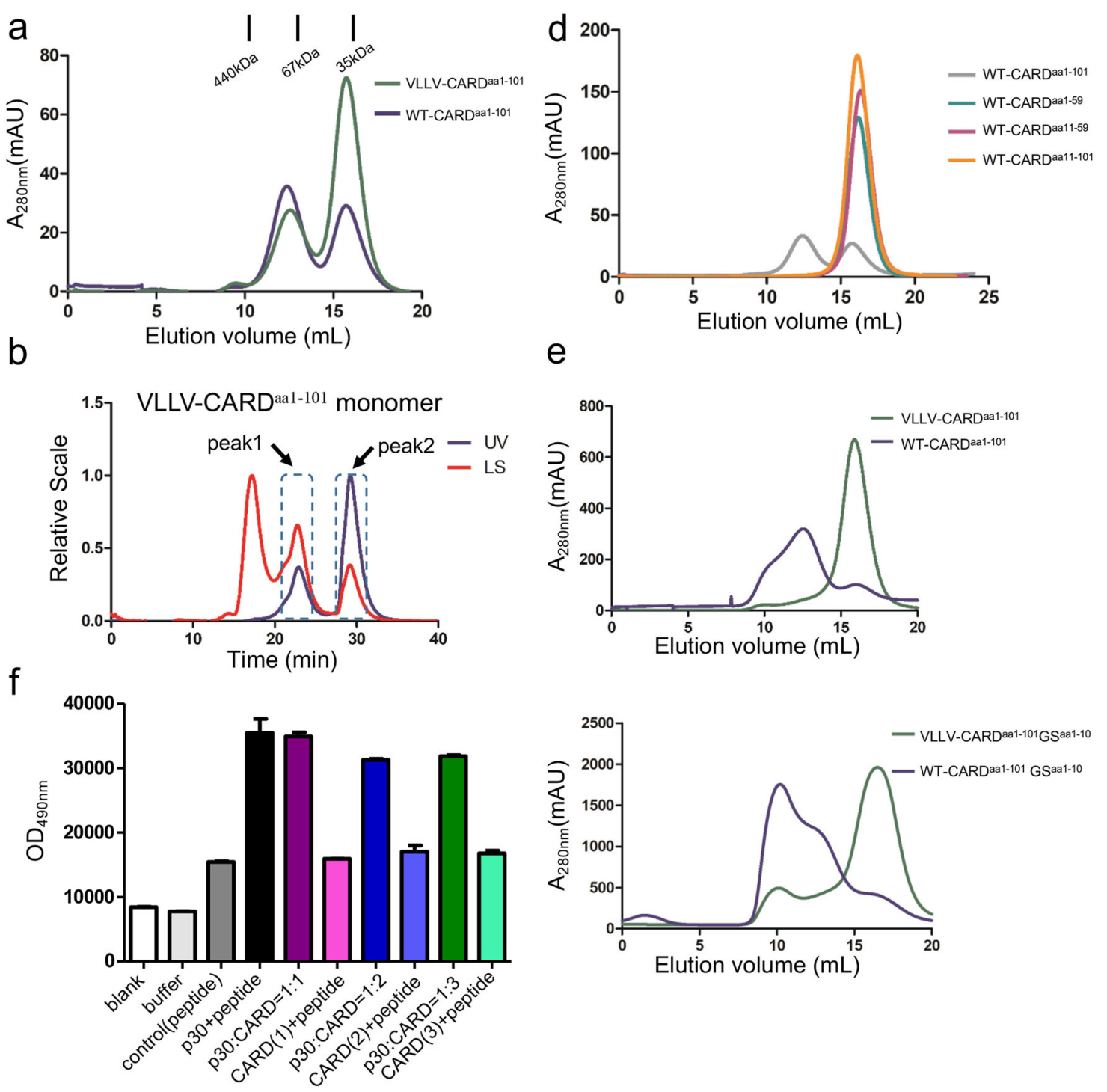

C

\begin{tabular}{ccc}
\hline Proteins(with MBP) & Peak1 MW(kDa) & Peak2 MW(kDa) \\
\hline $\begin{array}{c}\text { VLLV-CARD } \\
\text { monomer-101 }\end{array}$ & $258.8 \pm 1.1$ & $55.7 \pm 0.2$ \\
\hline
\end{tabular}

Fig. 6 Tetramerization of caspase-11 CARD ${ }^{\text {aa1-101 }}$ in solution. a E. coli-expressed MBP-tagged WT-CARD ${ }^{\text {aal-101 }}$ and VLLV-CARD ${ }^{\text {aa1-101 }}$ protein both elute as two peaks. b Multi-angle light scattering (MALS) analysis of VLLV-CARD ${ }^{\text {aal-101 }}$ monomer peak in $\mathbf{a}$. c ASTRA analysis of the molecular weight of different peaks in $\mathbf{b}$. $\mathbf{d}$ Gel-filtration chromatography of truncated CARDs with the same quantity of proteins (1 mg). e Gel-filtration chromatography analysis of MBP-tagged WT-CARD ${ }^{\text {aa1-101 }}$ and VLLV-CARD ${ }^{\text {aal-101 }}$ with a GS repeat sequence. $\mathbf{f}$ CARD inhibition of p30 proteolytic activity was detected by the fluorescence derived from the GSDMD cleavage reporter. CARD(1) indicates the concentration ratio between the catalytic domain and CARD protein as $1: 1 ; \operatorname{CARD}(2)$ indicates $1: 2 ; \operatorname{CARD}(3)$ indicates $1: 3$.

antibody was obtained from ABclona (AC002). Horseradish peroxidase (HRP)-Goat anti-rabbit IgG (D110058) and HRP-Goat anti-mouse IgG (D110087) were purchased from BBI. Unlabeled ultrapure LPS from E. coli
O111:B4 was purchased from Invitrogen. LDH release kit (C0017) was obtained from Beyotime.

Cell culture products were obtained from HyClone and fetal bovine serum (FBS) was obtained from Gibco. 


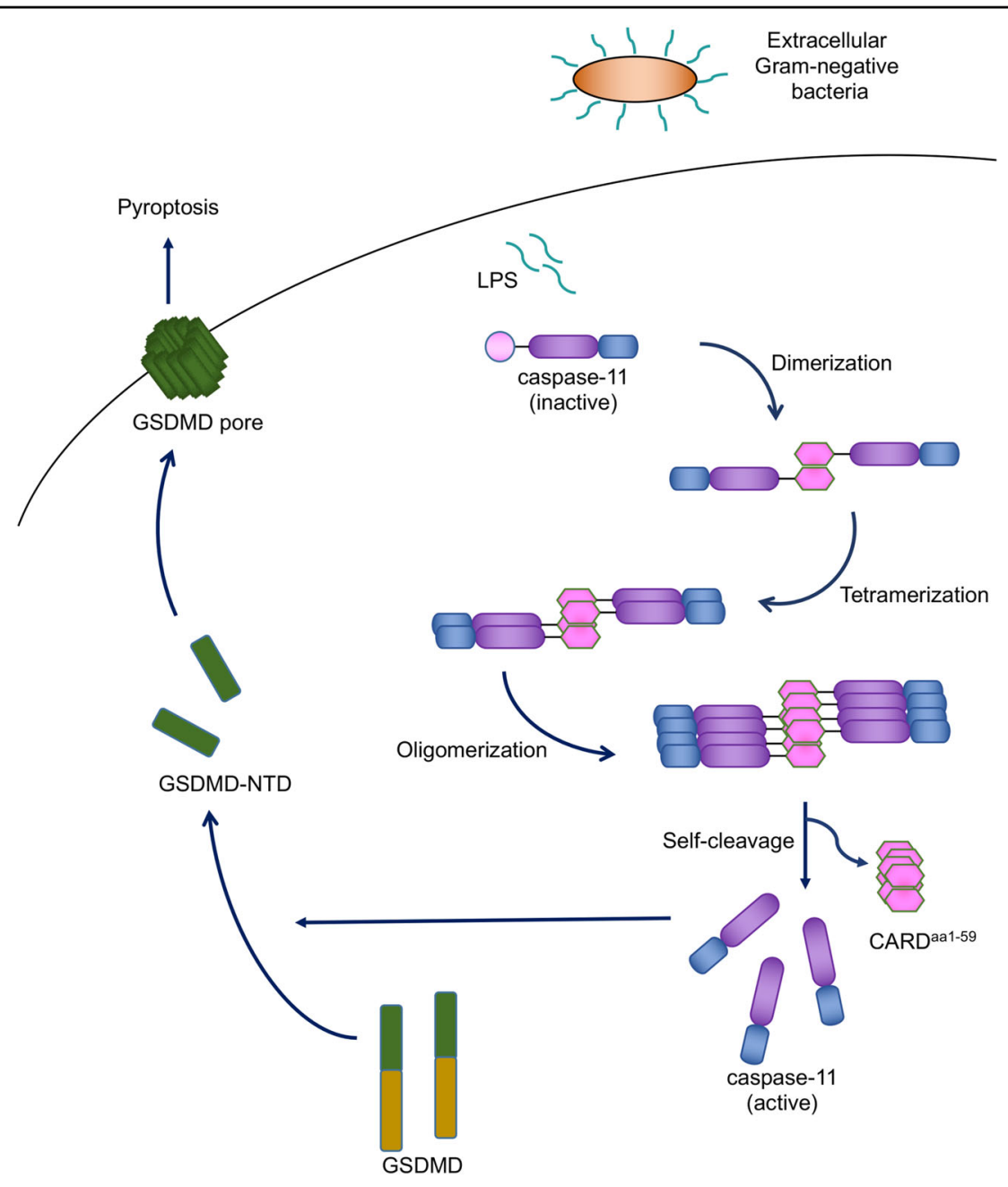

Fig. 7 Model of caspase-11 activation. LPS, a component of gram-negative bacteria, can be delivered into the cytosol of host cells. The presence of intracellular LPS results in the activation of the non-canonical inflammasome signaling pathway. The activation of caspase-11 requires the aggregation of CARD from a monomer, via a homodimer, to a homotetramer, then the oligomerization of caspase-11 induces self-cleavage, which results in the release of the catalytic domain of caspase-11. The catalytic domain of caspase-11 then cleaves GSDMD, inducing pyroptosis.

\section{Reverse transcription-quantitative PCR}

Quantification of caspase-4 expression in 293T cells was performed with SYBR Green I PCR Master Mix (Takara) in the StepOne Plus Real-Time PCR System (ABI). The primers used for each gene examined are listed below. CASP4: 5'-CAGACTCTATGCAAGAGAAGCAACGTATGGCA GGA-3' (forward) and 5'-CACCTCTGCAGGCCTGGACAATGATGAC-3' (reverse); actin: 5'-CATGTACGTTG CTATCCAGGC- $3^{\prime}$ (forward) and 5'-CTCCTTAATGTC ACGCACGAT-3' (reverse).

\section{In vitro caspase-11 protease activity assay}

The p20 and p10 fragments of the wild-type caspase- 11 were obtained through expression and the auto-processing of the full-length caspase-11 in E. coli. Synthetic self- quenched fluorescence peptide substrate DABYLGQLSLLSDGID-glu ( $2 \mu \mathrm{M}$, edans), which contains the caspase-11 cleavage site of GSDMD, was incubated with different concentrations of active caspase- 11 in $200 \mu \mathrm{L}$ buffer containing $150 \mathrm{mM} \mathrm{NaCl}, 1 \mathrm{mM}$ EDTA, $10 \mathrm{mM}$ dithiothreitol, and $50 \mathrm{mM}$ HEPES (pH 7.4). The reactions were incubated for $30 \mathrm{~min}$ at room temperature prior to the measurement of fluorescence intensity using a BIOTEK microplate reader (Synergy H1).

\section{Multi-angle light scattering}

MALS measurements were carried out on a DAWN HELEOS $8+$ instrument (Wyatt Technology) at room temperature. Caspase-11-CARD protein samples were diluted to $1 \mathrm{mg} / \mathrm{mL}$ in a buffer containing $250 \mathrm{mM} \mathrm{NaCl}$ 
and $10 \mathrm{mM}$ Tris- $\mathrm{HCl}(\mathrm{pH}$ 8.0) for analysis. The data were analyzed with the ASTRA software (Wyatt Technology).

\section{The sedimentation velocity analytical ultracentrifugation}

SV experiments were performed at 50,000 r.p.m. in a Proteomelab XL-A analytical ultracentrifuge (Beckman Coulter Instruments) at $20.0^{\circ} \mathrm{C}$ at a wave length of $280 \mathrm{~nm}$ (lower concentration) and $250 \mathrm{~nm}$ (high concentration) using $12 \mathrm{~mm}$ double-sector cells. Protein solution of different concentration were used in a buffer containing $250 \mathrm{mM} \mathrm{NaCl}$ and $10 \mathrm{mM}$ Tris- $\mathrm{HCl}$ (pH 8.0).

\section{Total internal reflection fluorescence microscopy}

HEK293T cells were transfected with plasmids encoding wild-type or mutant caspase-11. All cells were grown in growth media (high glucose Dulbecco's modified Eagle's medium with $10 \%$ FBS) and allowed to adhere overnight before transfection. Cells were analyzed $24 \mathrm{~h}$ after transfection. All samples were fixed with $4 \%$ paraformaldehyde in $1 \times$ phosphate-buffered saline for $15 \mathrm{~min}$ on ice. Samples were visualized with a Leica SR GSD microscope with a $\times 160$ oil objective and a $100 \mathrm{~nm}$ penetration depth.

\section{Statistical analysis}

Data were analyzed using Prism (GraphPad Software, version 5.01).

\section{Acknowledgements}

We thank the staff at GM/CA-CAT at the Advanced Photon Source (APS) and the BL17U1, BL18U, and BL19U1 beamline at Shanghai Synchrotron Radiation Facility (SSRF) for the assistance during data collection. T.J. is supported by the Strategic Priority Research Program of the Chinese Academy of Sciences (XDB29030104), the National Natural Science Foundation of China (31870731 and U1732109), the Fundamental Research Funds for the Central Universities, and the 100 Talents Program of The Chinese Academy of Sciences.
\end{abstract}

\section{Author details}

${ }^{1}$ Department of Obstetrics and Gynecology, The First Affiliated Hospital of USTC, Division of Life Sciences and Medicine, University of Science and Technology of China, No.17 Lujiang Rd, Hefei, Anhui 230001, China. ${ }^{2}$ Hefei National Laboratory for Physical Sciences at Microscale, CAS Key Laboratory of Innate Immunity and Chronic Disease, School of Basic Medical Sciences, Division of Life Sciences and Medicine, University of Science and Technology of China, Hefei, Anhui 230001, China. ${ }^{3}$ Hefei National Laboratory for Physical Sciences at the Microscale, Department of Chemical Physics, University of Science and Technology of China, Hefei, Anhui 230026, China. ${ }^{4}$ CAS Center for Excellence in Molecular Cell Science, Shanghai 200031, China

\section{Author contributions}

M.L. conceived, designed, performed most experiments, and wrote the paper. K.Z., Z.X., X.C., X.Y., S.F., and K.N. contributed to data collection and analysis. H.M. and W.Z. discussed the experiment results and contributed to the discussion. A.Z. contributed to manuscript writing. X.Y., B.L., Y.Z., R.Z., and T.J. contributed to design, supervision, and finalized the manuscript.

\section{Conflict of interest}

The authors declare that they have no conflict of interest.

\section{Publisher's note}

Springer Nature remains neutral with regard to jurisdictional claims in published maps and institutional affiliations.

Supplementary Information accompanies the paper at (https://doi.org/ 10.1038/s41421-020-00201-w).

Received: 21 March 2020 Accepted: 20 July 2020

Published online: 13 October 2020

\section{References}

1. Chen, H., Ning, X. \& Jiang, Z. Caspases control antiviral innate immunity. Cell. Mol. Immunol. 14, 736-747 (2017).

2. Broz, P. et al. Caspase-11 increases susceptibility to Salmonella infection in the absence of caspase-1. Nature 490, 288-291 (2012)

3. Case, C. L. et al. Caspase-11 stimulates rapid flagellin-independent pyroptosis in response to Legionella pneumophila. Proc. Natl Acad. Sci. USA 110 1851-1856 (2013)

4. Casson, C. N. et al. Caspase-11 activation in response to bacterial secretion systems that access the host cytosol. PLoS Pathog. 9, e1003400 (2013).

5. Rathinam, V. A. K. et al. TRIF licenses caspase-11-dependent NLRP3 inflammasome activation by gram-negative bacteria. Cell 150, 606-619 (2012).

6. Wang, S. et al. Murine caspase-11, an ICE-interacting protease, is essential for the activation of ICE. Cell 92, 501-509 (1998).

7. Hagar, J. A., Powell, D. A., Aachoui, Y., Ernst, R. K. \& Miao, E. A. Cytoplasmic LPS activates caspase-11: implications in TLR4-independent endotoxic shock. Science 341, 1250-1253 (2013).

8. Kayagaki, N. et al. Noncanonical inflammasome activation by intracellular LPS independent of TLR4. Science (80) 341, 1246-1249 (2013).

9. Kayagaki, N. et al. Non-canonical inflammasome activation targets caspase-11. Nature 479, 117-121 (2011)

10. Lee, B. L. et al. Caspase-11 auto-proteolysis is crucial for noncanonical inflammasome activation. J. Exp. Med. 215, 2279-2288 (2018).

11. Shi, J. et al. Inflammatory caspases are innate immune receptors for intracellular LPS. Nature 514, 187-192 (2014).

12. Kayagaki, N. et al. Caspase-11 cleaves gasdermin D for non-canonical inflammasome signalling. Nature 526, 666-671 (2015).

13. Shi, J. et al. Cleavage of GSDMD by inflammatory caspases determines pyroptotic cell death. Nature 526, 660-665 (2015).

14. Wang, S. Y. et al. Identification and characterization of Ich-3, a member of the interleukin-1-beta converting enzyme (Ice) Ced-3 family and an upstream regulator of ice. J. Biol. Chem. 271, 20580 (1996).

15. Lamkanfi, M. \& Dixit, V. M. Inflammasomes and their roles in health and disease. Annu. Rev. Cell Dev. Biol. https://doi.org/10.1146/annurev-cellbio101011-155745 (2012).

16. Riedl, S. J. \& Salvesen, G. S. The apoptosome: signalling platform of cell death Nat. Rev. Mol. Cell Biol. 8, 405-413 (2007).

17. Yin, X. et al. Crystal structure and activation mechanism of DR3 death domain. FEBS J. 286, 2593-2610 (2019).

18. Shen, C. et al. Molecular mechanism for NLRP6 inflammasome assembly and activation. Proc. Natl Acad. Sci. USA 116, 2052-2057 (2019).

19. Park, H. H. et al. The death domain superfamily in intracellular signaling of apoptosis and inflammation. Annu. Rev. Immunol. 25, 561-586 (2007).

20. Krissinel, E. \& Henrick, K. Inference of macromolecular assemblies from crystalline state. J. Mol. Biol. 372, 774-797 (2007).

21. Chu, L. H. et al. The oxidized phospholipid oxPAPC protects from septic shock by targeting the non-canonical inflammasome in macrophages. Nat. Commun. 9, 996 (2018).

22. Scott, F. L. et al. The Fas - FADD death domain complex structure unravels signalling by receptor clustering. Nature 457, 1019-1022 (2009).

23. Jin, T. et al. Design of an expression system to enhance MBP-mediated crystallization. Sci. Rep. 7, 40991 (2017).

24. Martinon, F. \& Tschopp, J. Inflammatory caspases and inflammasomes: master switches of inflammation. Cell Death Differ. 14, 10-22 (2007). 
25. Wang, Q. S. et al. Upgrade of macromolecular crystallography beamline BL17U1 at SSRF. Nud. Sci. Tech. 29, 68 (2018).

26. Otwinowski, Z. \& Minor, W. Processing of X-ray diffraction data collected in oscillation mode. Methods Enzymol. 276, 307-326 (1997).

27. Kabsch, W. et al. XDS. Acta Crystallogr. Sect. D. Biol. Crystallogr. 66, 125-132 (2010).
28. Adams, P. D. et al. PHENIX: a comprehensive Python-based system for macromolecular structure solution, https://doi.org/10.1107/ 97809553602060000865 (2012).

29. Jin, T., Huang, M., Smith, P., Jiang, J. \& Xiao, T. S. Structure of the caspaserecruitment domain from a zebrafish guanylate-binding protein. Acta Crystallogr. Sect. F. Struct. Biol. Cryst. Commun. 69, 855-860 (2013). 